\title{
Creating new $\beta$ cells: cellular transmutation by genomic alchemy
}

\author{
Larry G. Moss
}

Duke University Medical Center, Stedman Research Center, Durham, North Carolina, USA.

\begin{abstract}
To address insulin insufficiency, diabetes research has long focused on techniques for replacing insulin-producing $\beta$ cells. Studies in mice have suggested that, under some conditions, $\alpha$ cells possess the capacity to transdifferentiate into $\beta$ cells, although the mechanisms that drive this conversion are unclear. In this issue, Bramswig et al. analyzed the methylation states of purified human $\alpha, \beta$, and acinar cells and found $\alpha$ cells exhibit intrinsic phenotypic plasticity associated with specific histone methylation profiles. In addition to expanding our understanding of this potential source of $\beta$ cells, this compendium of carefully generated human gene expression and epigenomic data in islet cell subtypes constitutes a truly valuable resource for the field.
\end{abstract}

\section{Introduction}

The critical step that results in clinically manifested diabetes mellitus is loss (in type 1 diabetes due to autoimmune destruction) or deterioration (as in type 2 diabetes) of the functional pancreatic $\beta$ cell mass required to meet the body's demands for insulin. Understandably, a central goal in diabetes research has been to uncover strategies that could result in the replenishment of these cells. Whether the basic therapeutic approach might be to transplant replacement $\beta$ cells grown ex vivo or to induce new $\beta$ cell formation in vivo, an appropriate starting cell source must be identified and acceptable manipulations developed to produce normally functioning tissue.

While built on the extensive trove of knowledge of embryonic pancreatic islet development and the specific differentiation of $\beta$ cells, most approaches have relied on best-guess trial and error tactics. This applies to both the cell target and the intervention employed. Amazingly, a number of cell and tissue types have been successfully induced to express insulin and exhibit many $\beta$ cell characteristics (1) both in vitro (mouse and human embryonic stem cells, ref. 2) and in vivo (in mouse liver, refs. 3, 4; intestine, ref. 5; pancreatic exocrine, ref. 6; and glucagonproducing islet $\alpha$ cells, refs. 7-9). In mice, lineage tracing has confirmed that near total ablation of the $\beta$ cell population can induce

Conflict of interest: The author has declared that no conflict of interest exists.

Citation for this article: J Clin Invest. doi:10.1172/ JCI68348. transdifferentiation of $\alpha$ cells to a $\beta$ cell phenotype (7). This was a somewhat unexpected finding because an earlier lineage-tracing study showed that, during development, $\beta$ cells do not arise from glucagon-expressing progenitors (10). The $\alpha$ to $\beta$ phenotype switch can also be elicited by transgenic misexpression in $\alpha$ cells of a single protein, Pax4, a transcription factor required for $\beta$ cell formation during development (8).

However, key questions remain unanswered. What is it about a particular cell type that makes it amenable to reprogramming or transdifferentiation (plasticity) to a $\beta$ cell phenotype? Can specific interventions be identified and optimally matched to a given target cell for maximal efficacy? There are numerous levels and modalities at work acting in concert that determine the activity of a gene, including large-scale chromatin structure, gene accessibility, DNA methylation, posttranslational modifications of histones, and the function of transcription factors and their accessory proteins. Our growing understanding of these complex epigenetic and transcriptional mechanisms reveals an elegantly orchestrated process that controls gene transcription during development, differentiation, and regeneration.

Major inroads have been made into characterizing the transcriptional regulatory landscape of the genome in the context of whole islets (11-13). Moreover, recent studies in mice have examined the expressed transcriptome of $\beta$ cell-enriched islet cell populations that were isolated by cell sorting on the basis of scattering/flavin adenine dinucleotide (FAD) fluorescence (14) or insulin promoter-driven GFP (15). In the former study by the Ferrer group (14), whole genome mapping of individual histone methylation marks that correlate with active (H3K4me3) and repressed (H3K27me3) genes was performed in ES cells, embryonic pancreas, whole islets, pancreatic exocrine, and other tissue types. That group concluded that, in the process of development, $\beta$ cells acquired gene expression and active chromatin profiles most resembling neural tissues, consistent with their functional phenotype. However, the $\beta$ cell profile of Polycomb-mediated repressive marks was most closely related to those of exocrine pancreas and liver, which was proposed to reflect their common endodermal origins (14). Importantly, the Ferrer group did not examine characteristics of the $\alpha$ cell population in that study, and it is this topic that is the focus of the work in this issue by Bramswig et al., a collaborative effort of the Grompe and Kaestner labs (16).

\section{Inherent plasticity of $\alpha$ cells?}

The basis of this study is the capacity to FACS isolate enriched populations of human pancreatic islet $\alpha$ cells, $\beta$ cells, and exocrine (duct and acinar) cells (17, 18). Cell-type-specific discrimination was established by comparisons of gene expression profiles using RNA sequencing (RNASeq) of each fraction. Genome histone methylation profiles of $\mathrm{H} 3 \mathrm{~K} 4 \mathrm{me} 3$ and H3K27me3 marks were analyzed from each sample by ChIP/ultra high-throughput sequencing (ChIP-Seq). Genes were scored for histone methylation occurring in one of four patterns: monovalent $\mathrm{H} 3 \mathrm{~K} 4 \mathrm{me} 3$ (associated with active promoters), monovalent H3K27me3 (associated with polycomb-repressed genes), bivalent H3K4me3 and H3K27me3, or the absence of histone methylation. As expected, emblematic $\alpha$ cell-specific genes (e.g., glucagon) were marked only by H3K4me3 in $\alpha$ cells and by repression-associated $\mathrm{H} 3 \mathrm{~K} 27 \mathrm{me} 3$ in other cell types. $\beta$ Cell-specific genes (e.g., insulin) were similarly marked. However, 
$\beta$ cell
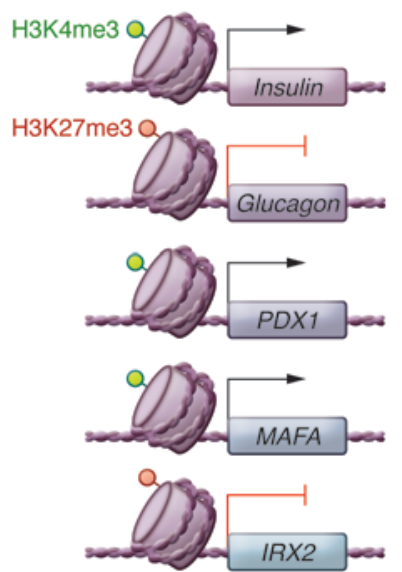

$\alpha$ cell
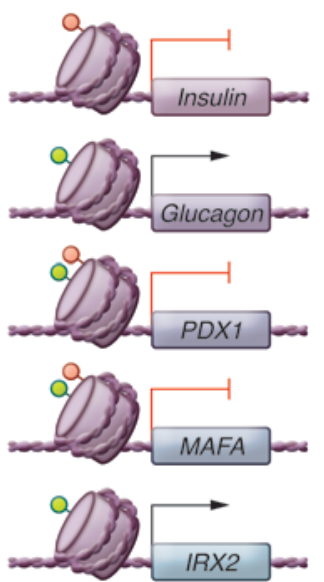

\section{Figure 1}

Asymmetry of $\beta$ and $\alpha$ cell histone methylation. In $\beta$ cells, most $\beta$ cell-specific genes (e.g., insulin) are monovalently marked as active by histone H3K4me3 (green), whereas $\alpha$ cellspecific genes (e.g., glucagon) are marked as repressed by histone H3K27me3 (red). The converse largely applies to $\alpha$ cells; however, Bramswig et al. show that several genes specifically required for $\beta$ cell differentiation (e.g., $P D X 1, M A F A$ ) are bivalently marked in $\alpha$ cells by $\mathrm{H} 3 \mathrm{~K} 4 \mathrm{me} 3$ and $\mathrm{H} 3 \mathrm{~K} 27 \mathrm{me} 3$, indicating a paused state with potential for activation. This potential does not appear to apply to $\alpha$ cell development genes in $\beta$ cells. the authors found that the great majority of genes with monovalent $\mathrm{H} 3 \mathrm{~K} 4 \mathrm{me} 3$ or monovalent $\mathrm{H} 3 \mathrm{~K} 27$ me 3 marks were not cell-type restricted, but common to all cell types analyzed, consistent with the results from the human genome-wide ENCODE study, which showed that for a large fraction of genes, most cell types share a common activity status (19).

However, the story takes an important turn when the bivalently marked genes were assessed. Bivalent $\mathrm{H} 3 \mathrm{~K} 4 \mathrm{me} 3$ plus $\mathrm{H} 3 \mathrm{~K} 27 \mathrm{me} 3$ marked genes are found at increased frequency in pluripotent stem cells and in developing embryos (20-22). While not rigorously proven, the general consensus is that such genes are associated with phenotypic plasticity, and while inactive, they are poised for rapid activation via a derepression event. In the above-cited study by the Ferrer group, a conversion of bivalent marks in ES cells and embryonic pancreas to monovalent marks in isolated adult $\beta$ cells was found for a subset of "neural-related" genes (14). Bramswig et al. found that $\alpha$ cells harbored many more bivalently marked genes than either $\beta$ or exocrine cells. Of the bivalently marked genes in $\alpha$ cells, roughly half were monovalently marked (by either H3K4me3 or $\mathrm{H} 3 \mathrm{~K} 27 \mathrm{me} 3)$ in $\beta$ cells. In the reverse comparison, only a quarter of $\beta$ cell bivalent genes were monovalently tagged in $\alpha$ cells. Many more bivalently marked genes in $\alpha$ cells than in $\beta$ cells were associated with developmental processes or transcriptional regulation. Finally, when genes with known roles unique to mature $\alpha$ cells or specific for $\alpha$ cell development were considered, most were marked by $\mathrm{H} 3 \mathrm{~K} 4 \mathrm{me} 3$ in $\alpha$ cells and monovalently (mainly by H3K27me3) in $\beta$ cells. When the converse comparison was made, most $\beta$ cell-specific function and development genes were $\mathrm{H} 3 \mathrm{~K} 4 \mathrm{me} 3$ marked in $\beta$ cells, but the majority of these were bivalently marked in $\alpha$ cells (ref. 16 and Figure 1).

Taken together, these data were interpreted as supporting the conclusion that on the basis of histone methylation profiles, $\alpha$ cells should have greater potential for plasticity than $\beta$ cells (or exocrine cells), possibly contributing to the capacity for $\alpha$ to $\beta$ transdifferentiation when the appropriate stimuli are applied. This work sets the stage for direct tests of this principle, including the demonstration of how Pax 4 can induce $\alpha$ to $\beta$ cell conversion. Bramswig et al. found that Pax 4 was not expressed in mature $\alpha$ or $\beta$ cells, and its gene was monovalently marked with repressive $\mathrm{H} 3 \mathrm{~K} 27 \mathrm{me} 3$ in both cell types. A predicted outcome of Pax 4 overexpression in islets would be a loss of the H3K27me3 mark on bivalently marked " $\beta$ cell genes" in the $\alpha$ cell population, accompanied by an increase in transcription. Similar approaches could be taken for other cell types that can be transdifferentiated into $\beta$-like cells to uncover common themes that define plasticity and potential for $\beta$ cell differentiation.

\section{Limitations of this approach}

Histone methylation patterns are associated with gene expression activity, but are not absolute determinants that can be applied as robust, unbiased predictors of gene activity. For example, it is known that a subset of genes marked by only $\mathrm{H} 3 \mathrm{~K} 4 \mathrm{me} 3$ are not transcriptionally active $(23,24)$ Indeed, Bramswig et al. found that a large fraction of the monovalently H3K4me3 marked genes were not expressed (16). This may be due to other mechanisms involved in regulation of gene activity, including the presence of $\mathrm{CPG}$ islands, higher order chromatin structure, DNA methylation, other posttranslational modifications of histones, or individual transcription factors. On the other hand, the monovalent $\mathrm{H} 3 \mathrm{~K} 27$ me 3 mark is very tightly associated with a repressed state (25). The "ready" status of bivalently marked genes is an assumption based on association. Rigorously determining whether a given bivalent mark is equivalent to a poised state would require establishing whether RNA pol II was poised or paused at the appropriate location, ready for release and transcriptional elongation, a highly focused and technically challenging experiment (26).

Another significant caveat to the interpretation of bivalent marks in this study could alter the conclusions: the presence of more than one cell type in a sample. Bramswig et al. isolated $\alpha$ cell-enriched fractions, but these were likely not pure populations. The RNA-Seq data suggested that the $\beta$ cell-enriched sample included a significant number of somatostatin-expressing $\delta$ cells, while the $\alpha$ cell fraction contained PPY-expressing cells. If in one cell type, a given gene bears only $\mathrm{H} 3 \mathrm{~K} 4 \mathrm{me} 3$ and in the other cell type it is $\mathrm{H} 3 \mathrm{~K} 27 \mathrm{me} 3$-marked, separate $\mathrm{H} 3 \mathrm{~K} 4 \mathrm{me} 3$ and $\mathrm{H} 3 \mathrm{~K} 27 \mathrm{me} 3 \mathrm{ChIP}$ analysis would suggest bivalency. Similarly, heterogeneity among the $\alpha$ cell population, as has been shown for $\beta$ cells (27), might 
also be interpreted as bivalency in this study. In either case, competence for $\alpha$ to $\beta$ transdifferentiation could be due to factors other than true histone methylation bivalency, such as a subset of $\alpha$ cells that are competent to undergo conversion to $\beta$ cells.

This question might be addressed by assessing presence of both histone marks on a single allele by sequential Chip (so called Chip-reChip), wherein the chromatin is first precipitated with an antibody against one histone mark and then reprecipitated with an antibody to the second mark (28). Indeed, this exact approach has been used to demonstrate $\mathrm{H} 3 \mathrm{~K} 4 \mathrm{me} 3 / \mathrm{H} 3 \mathrm{~K} 27 \mathrm{me} 3$ bivalency of genes during a key step in the differentiation of embryonic stem cells to insulinexpressing endocrine cells (29).

The confounding issue of cellular heterogeneity will present a major challenge for the next phase of the ENCODE human genome study as analysis moves from cell lines to native tissues (19). In the near future, further refinement of reagents are expected; this will provide higher purity enrichment of individual cell types. Moreover, improvements in techniques will reduce required sample sizes, allowing for simultaneous analysis of multiple epigenetic marks and other types of data to significantly enhance predictive power.

\section{Methylation as the alchemist's target}

To more directly tie methylation status to gene expression, Bramswig et al. treated intact human islets with Adox, a nonspecific methylation inhibitor, which is known to decrease $\mathrm{H} 3 \mathrm{~K} 27$ me3 levels. Adox treatment resulted in a decrease in $\mathrm{H} 3 \mathrm{~K} 27 \mathrm{me} 3$ at the $\beta$ cell-specific genes PDX1 and MAFA. Because these genes are monovalently marked with $\mathrm{H} 3 \mathrm{~K} 4 \mathrm{me} 3$ in $\beta$ cells and bivalently marked in $\alpha$ cells, the decline represents derepression in $\alpha$ cells. A striking finding was that, when islets were examined by immunofluorescence, double glucagon/insulin staining cells could be visualized in treated but not untreated islets. Treated and untreated islets were then sorted by cell type and subjected to RNA-Seq analysis. They found overall changes in expression, suggesting that $\alpha$ cells had become more $\beta$-like, but $\beta$ cells were not more $\alpha$-like (16).

Thus Bramswig et al. have demonstrated that histone methylation is involved in $\alpha$ to $\beta$ cell conversion and that the phenotype of $\alpha$ cells is plastic and amenable to pharmacological intervention. Furthermore, these results may help explain the surprising finding that Glucagon-cre-targeted knockout of menin in $\alpha$ cells leads to insulinoma formation (30), as one proposed activity of menin is the modulation of the Mll family of histone methylases (31).

\section{Clinical implications}

There is an obvious interest in the possibility of exploiting $\alpha$ cells as a source of replacement $\beta$ cells. For in vivo interventions to reach the bedside, pharmacological tools for triggering this targeted transdifferentiation pathway will need to be developed. As discussed above, the findings of Bramswig et al. point to a methylation-sensitive step that could be a drug target, especially as more specific methylase inhibitors become available. Alternatively, progress is being made in the development of pharmacologic inhibitors of the histone mark readers, the multiprotein complexes that are responsible for recognizing a specific mark and bringing about transcriptional activation or repression (32). There are already early phase studies describing high throughput screens for compounds that can induce insulin expression in $\alpha$-like cell lines (33).

An intriguing finding in the $\alpha$ cell Pax 4 overexpression mouse study is that the $\alpha$ to $\beta$ cell conversion does not result in a loss of $\alpha$ cells because it is accompanied by an extensive hyperplasia of glucagon-expressing cells (8). It has been known for some time that knockout of the glucagon receptor or administration of glucagon receptor inhibitors results in $\alpha$ cell hyperplasia and even tumor development $(34,35)$. The Drucker lab recently demonstrated that mice with liver-specific knockout of the glucagon receptor also experience $\alpha$ cell hyperplasia (36), suggesting that a circulating factor may drive this response, which if isolated could lead to the development of drugs to expand $\alpha$ cell mass. Perhaps diabetes therapy could be as simple as 2 swings of the alchemist's wand, the first expanding the endogenous islet $\alpha$ cell population and the second inducing conversion to functioning $\beta$ cells.

Thus, Bramswig et al. show a way past the experimental barrier of islet cellular heterogeneity and provide a valuable resource to rationally design novel approaches for the generation of $\beta$ cells by epigenetic reprogramming.

\section{Acknowledgments}

Thanks to Christopher B. Newgard for critical reading of the manuscript.
Address correspondence to: Larry G. Moss, Duke University Medical Center, Stedman Research Center, Box 3937, Durham, North Carolina 27710, USA. Phone: 617.479.2310; Fax: 919.477.0632; E-mail: larry.moss@ duke.edu.

1. Willet S, Wright C. Pancreatic plasticity and reprogramming: novel directions towards disease therapy. In: Ainscough J, Yamanaka S, Tada T, eds. Nuclear Reprogramming and Stem Cells. New York, New York, USA: Humana Press; 2011:193-215.

2. Kroon E, et al. Pancreatic endoderm derived from human embryonic stem cells generates glucoseresponsive insulin-secreting cells in vivo. Nat Biotechnol. 2008;26(4):443-452.

3. Kojima H, et al. NeuroD- $\beta$ cellulin gene therapy induces islet neogenesis in the liver and reverses diabetes in mice. Nat Med. 2003;9(5):596-603.

4. Ferber $S$, et al. Pancreatic and duodenal homeobox gene 1 induces expression of insulin genes in liver and ameliorates streptozotocin-induced hyperglycemia. Nat Med. 2000;6(5):568-572.

5. Talchai C, Xuan S, Kitamura T, DePinho RA, Accili D. Generation of functional insulin-producing cells in the gut by Foxo1 ablation. Nat Genet. 2012; 44(4):406-412.

6. Zhou Q, Brown J, Kanarek A, Rajagopal J, Melton DA. In vivo reprogramming of adult pancreatic exocrine cells to $\beta$-cells. Nature. 2008;455(7213):627-632.

7. Thorel F, et al. Conversion of adult pancreatic $\alpha$-cells to $\beta$-cells after extreme $\beta$-cell loss. Nature. 2010;464(7292):1149-1154

8. Collombat P, et al. The Ectopic Expression of $\mathrm{Pax} 4$ in the mouse pancreas converts progenitor cells into $\alpha$ and subsequently $\beta$ cells. Cell. 2009; 138(3):449-462.

9. Chung CH, Hao E, Piran R, Keinan E, Levine F. Pancreatic $\beta$-cell neogenesis by direct conversion from mature $\alpha$-cells. Stem Cells. 2010;28(9):1630-1638.

10. Herrera PL. Adult insulin- and glucagon-producing cells differentiate from two independent cell lineages. Development. 2000;127(11):2317-2322.

11. Bhandare R, et al. Genome-wide analysis of histone modifications in human pancreatic islets. Genome Res. 2010;20(4):428-433.

12. Stitzel ML, et al. Global epigenomic analysis of primary human pancreatic islets provides insights into type 2 diabetes susceptibility loci. Cell Metab. 2010;12(5):443-455.

13. Tennant BR, et al. Identification and analysis of murine pancreatic islet enhancers [published online ahead of print December 14, 2012]. Diabetologia. doi:10.1007/s00125-012-2797-5.

14. van Arensbergen J, et al. Derepression of Polycomb targets during pancreatic organogenesis allows insulin-producing $\beta$-cells to adopt a neural gene activity program. Genome Res. 2010;20(6):722-732.

15. Ku GM, et al. Research resource: RNA-Seq reveals unique features of the pancreatic $\beta$-cell transcriptome. Mol Endocrinol. 2012;26(10):1783-1792.

16. Bramswig NC, et al. Epigenomic plasticity enables human pancreatic $\alpha$ to $\beta$ cell reprogramming. J Clin Invest. 2013;123(3):1275-1284.

17. Dorrell C, Abraham SL, Lanxon-Cookson KM, Canaday PS, Streeter PR, Grompe M. Isolation of major pancreatic cell types and long-term cultureinitiating cells using novel human surface markers. Stem Cell Research. 2008;1(3):183-194.

18. Dorrell C, et al. Transcriptomes of the major human pancreatic cell types. Diabetologia. 2011; 54(11):2832-2844.

19. ENCODE Project Consortium, et al. An integrated encyclopedia of DNA elements in the human genome. Nature. 2012;489(7414):57-74.

20. Bernstein BE, et al. A bivalent chromatin structure 
marks key developmental genes in embryonic stem cells. Cell. 2006;125(2):315-326.

21. Vastenhouw NL, Schier AF. Bivalent histone modifications in early embryogenesis. Curr Opin Cell Biol. 2012;24(3):374-386

22. Pan G, et al. Whole-genome analysis of histone $\mathrm{H} 3$ lysine 4 and lysine 27 methylation in human embryonic stem cells. Cell Stem Cell. 2007;1(3):299-312.

23. Guenther MG, Levine SS, Boyer LA, Jaenisch R, Young RA. A chromatin landmark and transcription initiation at most promoters in human cells. Cell. 2007;130(1):77-88.

24. Shi X, et al. ING2 PHD domain links histone H3 lysine 4 methylation to active gene repression. Nature. 2006;442(7098):96-99.

25. Greer EL, Shi Y. Histone methylation: a dynamic mark in health, disease and inheritance. Nat Rev Genet. 2012;13(5):343-357.

26. Levine M. Paused RNA polymerase II as a developmental checkpoint. Cell. 2011;145(4):502-511.

27. Katsuta H, et al. Subpopulations of GFP-marked mouse pancreatic $\beta$-cells differ in size, granularity, and insulin secretion. Endocrinology. 2012; 153(11):5180-5187.

28. Truax AD, Greer SF. ChIP and Re-ChIP assays: investigating interactions between regulatory proteins, histone modifications, and the DNA sequences to which they bind. Methods Mol Biol. 2012;809:175-188

29. Xie R, et al. Dynamic chromatin remodeling mediated by polycomb proteins orchestrates pancreatic differentiation of human embryonic stem cells. Cell Stem Cell. 2013;pii:S1934-5909(12)00706-0.

30 . Lu J, et al. $\alpha$ cell-specific Men 1 ablation triggers the transdifferentiation of glucagon-expressing cells and insulinoma development. Gastroenterology. 2010;138(5):1954-1965.e1958.

31. Agarwal SK, Jothi R. Genome-wide characterization of menin-dependent $\mathrm{H} 3 \mathrm{~K} 4 \mathrm{me} 3$ reveals a specific role for menin in the regulation of genes implicated in MEN1-like tumors. PLoS One. 2012 7(5):e37952.
32. James LI, et al. Discovery of a chemical probe for the L3MBTL3 methyllysine reader domain [published online ahead of print January 6, 2013]. Nat Chem Biol. doi:10.1038/nchembio.1157.

33. Fomina-Yadlin D, et al. Small-molecule inducers of insulin expression in pancreatic-cells. Proc Natl Acad SciU S A. 2010;107(34):15099-15104.

34. Gu W, et al. Long-term inhibition of the glucagon receptor with a monoclonal antibody in mice causes sustained improvement in glycemic control, with reversible alpha-cell hyperplasia and hyperglucagonemia. J Pharmacol Exp Ther. 2009;331(3):871-881.

35. Gelling RW. Lower blood glucose, hyperglucagonemia, and pancreatic $\alpha$ cell hyperplasia in glucagon receptor knockout mice. Proc Natl Acad Sci U S A. 2003;100(3):1438-1443

36. Longuet $\mathrm{C}$, et al. Liver-specific disruption of the murine glucagon receptor produces $\alpha$-cell hyperplasia: evidence for a circulating $\alpha$-cell growth factor [published online ahead of print November 16 2012]. Diabetes. doi:10.2337/db11-1605.

\title{
Virgin birth: engineered heart muscle from parthenogenetic stem cells
}

\author{
Sara J. McSweeney and Michael D. Schneider
}

British Heart Foundation Centre of Research Excellence, National Heart and Lung Institute, Imperial College London, London, United Kingdom.

\begin{abstract}
Cardiac muscle restitution, or true regeneration, is an unmet need in the treatment of myocardial infarction (MI), prompting a decade of study with stem cells of many kinds. Among key obstacles to effective cardiac cell grafting are the cost of autologous stem cell-derived cardiomyocytes, the ethical implications of using embryonic stem cell (ESC) products, immunological barriers to allogeneic cells, functional maturation beyond just the correct lineage decision, and the lack of durable engraftment. In this issue of the JCI, Didié and colleagues show that cardiomyocytes made from parthenogenetic stem cells (PSCs) and deployed as engineered heart muscle (EHM) may overcome all of these formidable barriers.
\end{abstract}

Survival after MI has increased dramatically over the last 30 years, chiefly due to improvements in restoring blood flow to the ischemic heart and in preventing longterm dilation and wall thinning. However, these remedies do not address the underlying cause of biomechanical dysfunction after damage, namely the death of up to 1 billion cardiomyocytes (1). Under normal circumstances, myocyte replacement is measurable but scant (2). Therefore, strategies are being developed to replace the lost cardiomyocytes using various types of stem or progenitor cells. Clinical investigations of bone marrow populations including mesenchymal stem cells have shown

Conflict of interest: The authors have declared that no conflict of interest exists.

Citation for this article: J Clin Invest. doi:10.1172/ JCI67961. encouraging, though limited, benefits and are currently in phase III trials; however, these are now envisioned as working chiefly through angiogenesis and paracrine effects, not myocyte replacement (3). Heart-derived progenitor cells with clearer potential for cardiac muscle creation have recently completed phase I safety trials (4).

Pluripotent cells with the capacity to generate all the cell types of the body are an alternative strategy for heart repair that have long been studied in the laboratory, but have been slow to find their way into the clinic. Injection of pluripotent cells themselves is problematic due to their ability to form teratomas (5), so therapeutic use of these would require the rigorous purification of stem cell-derived cardiomyocytes or perhaps their committed precursors. Translation of such work to the clinic has also been hindered by many other issues regarding the use of human ESCs, including ethical disputes and the fundamental challenge of immunological rejection. For this reason, immunologically privileged approaches to generate heart muscle from pluripotent stem cells must be considered. Induced pluripotent stem cells (iPSCs) are readily created from skin fibroblasts or blood, and do not raise the ethical objections associated with ESCs. Like ESCs they can differentiate into cardiomyocytes, but can, in principle, be generated as a patient-specific therapy (6). However, the epigenetic memory of these cells could bias them toward certain fates (7), their immunological status has been questioned (6), and the logistics of "bespoke" therapy are far more complex than the hypothetical universal donor.

\section{Single-parent stem cells}

As a new option for cell therapy, Didié et al. show in the current study that PSCs could be an alternative to ESCs in cardiac regeneration (8), as they do not have the same ethical implications. Parthenogenesis (Greek for "virgin birth") is a natural form of asexual reproduction observed in plants, invertebrates, fish, amphibians, and reptiles. During the formation of a normal mammalian embryo, oocytes are arrested in metaphase II until fertilization, when 